\title{
Poly(ethylene glycol) Enclatherated Pectin-Mucin Submicron Matrices for Intravaginal Anti-HIV-1 Drug Delivery
}

Felix Mashingaidze ${ }^{1}$, Yahya E. Choonara ${ }^{1}$, Pradeep Kumar ${ }^{1}$, Lisa C. du Toit ${ }^{1}$, Vinesh Maharaj ${ }^{2}$, Eckhart Buchmann ${ }^{3}$ and Viness Pillay ${ }^{1 *}$

${ }^{1}$ Wits Advanced Drug Delivery Platform Research Unit, Department of Pharmacy and Pharmacology, School of Therapeutic Sciences, Faculty of Health Sciences, University of the Witwatersrand, Johannesburg, 7 York Road, Parktown, 2193, South Africa

${ }^{2}$ Department of Chemistry, University of Pretoria, Pretoria, 0002, South Africa

${ }^{3}$ Department of Obstetrics and Gynaecology, Faculty of Health Sciences, University of the Witwatersrand, Johannesburg, 7 York Road, Parktown, 2193, South Africa

\author{
*Corresponding Author: \\ Professor Viness Pillay \\ Tel: $+27-11-717-2274$ \\ Fax: $+27-11-642-4355$ \\ Fax2Email: +27-86-553-4733 \\ Email: viness.pillay@wits.ac.za
}

\section{Abstract}

This paper explores the potential of polyethylene glycol enclatherated pectin-mucin (PEGencl-PEC:MUC) submicron matrices (SMMs) as an intravaginal drug delivery system capable of delivering an anti-HIV-1 agent (zidovudine; AZT) over a prolonged duration. A three factor and three level $\left(3^{3}\right)$ Box-Behnken statistical design was employed to optimize the SMMs. Optimized PEG-encl-PEC:MUC SMMs prepared as a stable W/O emulsion (determined by the degree of reversible colloidal phenomena) were spherical with a mean particle size of $270.6 \pm 5.533 \mathrm{~nm}$ and mean zeta potential of $-34.4 \pm 0.539 \mathrm{mV}$. The microencapsulation of AZT and the hydrogen bonding mediated shielding of AZT by SMMs was confirmed by Fourier Transform Infrared (FTIR) analysis. The thermochemical (differential scanning calorimetry and thermogravimetric analysis) data proposed that $\mathrm{Ca}^{2+}$ based macromolecular ionic crosslinking as well as the intermolecular interactions may be responsible for the thermal stability of the delivery system. The partially amorphous nature of drug-loaded SMMs, as confirmed by X-ray diffraction patterns, further strengthened the matricization of AZT into the pectin-mucin matrix. In vitro drug release studies from the SMMs showed approximately $91 \%$ zidovudine release in simulated vaginal fluid (SVF) and $94 \%$ in phosphate buffered saline (PBS) in 24 hours. The mean dissolution time (MDT) of zidovudine from the SMMs was 5.974 hours. The attainment of required dimensional structure and drug release profiles from SMMs highlights the potential of their inclusion into a secondary carrier system for extended and controlled intravaginal stay. 
Keywords: Anti-HIV-1, zidovudine, pectin, mucin, poly(ethylene glycol), intravaginal delivery, submicron matrices.

\section{Graphical abstract:}
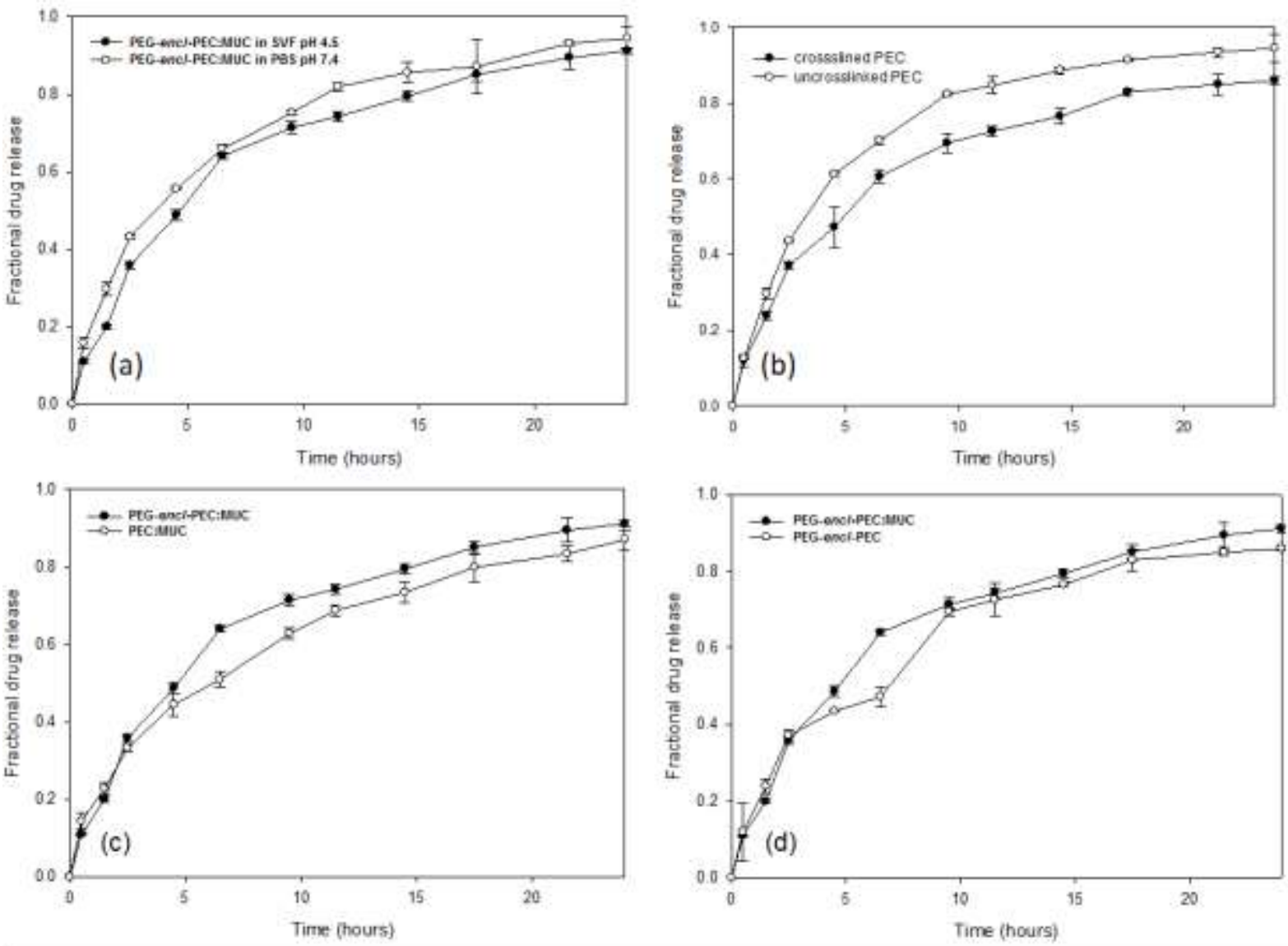

Comparison of AZT release from (a) PEG-enc/.PEC:MUC SMMs in SVF and PBS; (b) erosslinked and uncrosslinked PEC SMMs in SVF (c) PEG-encl-PEC:MUC and PEC-MUC SMMs in SVF; and (d) PEG-encl-PEC:MUC and PEC-PEG SMMs in SVF.

\section{Introduction}

Focus on preventing vaginal HIV-1 transmission has led to the development and usage of a physical polymeric barrier/sheath commonly known as a condom which is worn during coitus to prevent the mixing of male and female reproductive fluids thus preventing HIV-1 infection. Though largely effective in preventing HIV-1 transmission, the condom has been hampered by inappropriate, infrequent usage and to some extent it has suffered problems of cultural and religious acceptability. As an alternative to the condoms, contemporary research is being done on microbicides. These are anti-HIV-1 agents that may be applied topically to the vaginal or rectal cavity to prevent HIV-1 transmission (Stone, 2002; Woolfson et al., 2010). A keen interest in using antiretroviral drugs as microbicides has also emerged given their success in treating HIV/AIDS (Klaase et al., 2008). Although microbicides hold much 
promise and their concept is sound, clinical trials conducted up-to-date have failed to demonstrate efficacy (Hendrix et al., 2009). Several questions have been raised and some answers postulated to try end explain why anti-HIV-1 agents effective in vitro have failed to demonstrate efficacy in vivo. One common possible explanation given is microbicide formulations did not take into consideration and did not address some fundamental anatomical, physiochemical and physiological principles involved in vaginal HIV-1 transmission. Such factors include; the need to maintain or augment the vaginal mucosal barrier properties, maintenance of the HIV-1 prohibitive acidic $\mathrm{pH}(\sim 4.5)$ of the vaginal cavity, presence of the anti-HIV-1 agent in appropriate quantities in the vaginal cavity where it has to be distributed extensively and exhaustively on the vaginal mucosa, anti-HIV-1 agent presence in the sub-epithelia to counteract HIV-1 that would have transcytosed the vaginal epithelia and having the microbicide stays at the site for a longer duration than HIV-1 (Hendrix et al., 2009).

The development of topically applied chemical agents that may effectively prevent vaginal HIV-1 transmission (microbicides) has been a major challenge for pharmaceutical scientists (Hendrix et al., 2009; Adams and Kashuba, 2012; Pillay et al., 2012). The difficulties have included; microbicide acceptance as an alternative HIV-1 prevention modality, pharmacokinetic inadequacy whereby the microbicide has to be distributed throughout the vaginal mucosa in sufficient quantities to prevent HIV-1 transmission and mucosal toxicity which diminishes the vaginal mucosa's barrier properties resulting in easy HIV-1 mucosal transcytosis and transmission (Adams and Kashuba, 2012; Pillay et al., 2012). Despite having vast potential as a preventative tool against vaginal HIV-1 transmission, research conducted thus far has failed to provide an effective anti-HIV-1 microbicide product to the market. Various formulations have been prepared to try and mitigate these concerns, among them; gels, creams, films, tablets and rings (Hendrix et al., 2009). Gels, creams and films are indiscrete, sticky and are short acting thus require frequent application before and after every coital act whilst tablets and rings are discrete and may be formulated to deliver the anti-HIV-1 agent over an extended duration (Klaase et al., 2008). In this study, poly(ethylene glycol) enclatherated pectin-mucin submicron matrices (SMMs) were formulated for potential inclusion into a polymeric caplet eventually forming a composite polymeric drug delivery system as a potential microbicide drug delivery system. The submicron matrices were formulated from biocompatible materials; pectin, porcine gastric mucin and polyethylene glycol. These polymers formed the framework structure of the submicron matrices enabling the encapsulation and controlled release of zidovudine, the model antiretroviral used in this study. 


\section{Materials and Methods}

\subsection{Materials}

Commercial grade pectin (PEC), GENU ${ }^{\circledR}$ pectin type USP/100 [Degree of esterification (DE) 55-65\%] was obtained from CP Kelco ApS, Lille Skensved, Denmark. Porcine gastric mucin type III (MUC) with 1-1.5\% bound sailic acid, polyethylene glycol of $M_{w} 400$ (PEG) and Kollidon $^{\circledR} \mathrm{SR}$ (KSR) and phosphate buffered saline (PBS, pH 7.4) were purchased from Aldrich $^{\circledR}$ (Sigma-Aldrich Inc., St. Louis, USA). Poly (D,L-lactide) was purchased from Boehringer Ingelheim, Ingelheim, Germany. Carbopol 794P NF was obtained from Noveon Inc., Cleveland, OH, USA. The model anti-HIV-1 active pharmaceutical ingredient (API), zidovudine (AZT) was obtained from Glaxo Smith Kline, Middlesex, UK. Other materials and excipients including; calcium chloride, magnesium stearate (MS), glucose and cyclohexane were of analytical grade and were utilized as obtained. Simulated vaginal fluid (SVF, pH 4.5) was prepared from analytical grade reagents in accordance to Owen and Katz's formulation (Owen and Katz, 1999).

\subsection{Box-Behnken design approach for the preparation of PEG-encl-PEC:MUC SMMs}

A three-factor, three-level $\left(3^{3}\right)$ Box-Behnken statistical design on MINITAB ${ }^{\circledR}(\mathrm{V} 14$, State College, Pennsylvania, USA) was employed to optimize the preparation of AZT-loaded PEGencl-PEC:MUC SMMs (Karnachi and Khan, 1996; Box and Behnken, 1960). Upper and lower levels of three independent parameters that included; ultrasonication time (ST), surfactant concentration (SC) and drug:polymer (D:P) ratio were chosen due to their high significance in the fabrication of the SMMs. The dependent parameters or responses that comprised; particle size (PS), zeta potential (ZP) and mean dissolution time (MDT) were sought, as presented in Table 1. Fifteen formulations were generated from the Box-Behnken

Table 1: Independent parameters and responses from the Box-Behnken statistical design used to optimize AZTloaded PEC-MUC-PEG SMMs

\begin{tabular}{lcc}
\hline Independent parameter & \multicolumn{2}{c}{ Levels } \\
\cline { 2 - 3 } & Lower & Upper \\
ultrasonication time $(\mathrm{min})$ & 5 & 10 \\
surfactant concentration $\%(\mathrm{v} / \mathrm{v})$ & 1.5 & 2 \\
drug:polymer ratio & 0.5 & 1 \\
& & \\
Response & & Objective \\
particle size $(\mathrm{nm})$ & minimize \\
zeta potential $(\mathrm{mV})$ & minimize \\
mean dissolution time (hours) & & maximize \\
\hline
\end{tabular}


design (Table 2). These formulations were prepared and experimentally tested and the results obtained were fed into the MINITAB $^{\circledR}$ design software which then computed the optimized formulation's independent parameter and expected response values.

Table 2: Formulations generated using a Box-Behnken statistical design for the optimization of AZT-loaded PECMUC-PEG SMMs

\begin{tabular}{|c|c|c|c|}
\hline Formulation number & Ultrasonication time (min) & $\begin{array}{l}\text { Surfactant concentration } \\
\%(v / v)\end{array}$ & Drug:polymer ratio \\
\hline 1 & 10 & 1.75 & 0.5 \\
\hline 2 & 10 & 1.5 & 0.75 \\
\hline 3 & 5 & 1.5 & 0.75 \\
\hline 4 & 10 & 2 & 0.75 \\
\hline 5 & 5 & 2 & 0.75 \\
\hline 6 & 7.5 & 2 & 1 \\
\hline 7 & 5 & 1.75 & 1 \\
\hline 8 & 7.5 & 1.75 & 0.75 \\
\hline 9 & 7.5 & 1.75 & 0.75 \\
\hline 10 & 10 & 1.75 & 1 \\
\hline 11 & 7.5 & 1.5 & 0.5 \\
\hline 12 & 5 & 1.75 & 0.5 \\
\hline 13 & 7.5 & 1.5 & 1 \\
\hline 14 & 7.5 & 2 & 0.5 \\
\hline 15 & 7.5 & 1.75 & 0.75 \\
\hline
\end{tabular}

\subsection{Preparation of AZT- loaded PEG-encl-PEC:MUC SMMs}

AZT-loaded PEG-encl-PEC:MUC SMMs where prepared using a crosslinking-emulsion technique. PEC, MUC, PEG and AZT (360mg) were subsequently dispersed and dissolved in MilliQ water, obtained from a MilliQ ${ }^{\circledR}$ gradient water purification system (Millipore SAS, Molsheim, France), whilst stirring for $15 \mathrm{~min}$ to form an aqueous phase which was then crosslinked by drop-wise addition of calcium chloride. The PEC:MUC ratio used was 9:1, PEG was $0.1 \mathrm{mLs}$ and drug (AZT):polymer (MUC, PEC and PEG) ratios used were in accordance with the Box-Behnken-design. A water-in-oil (W/O) emulsion was prepared by ultrasonication using a high intensity ultrasonic processor (Sonics Vibracell VCX 130, Sonics Materials INC, Newtown, CT, USA) with the crosslinked PEG-encl-PEC:MUC-AZT dispersion acting as the aqueous phase and cyclohexane as the oil phase. The w/o ratio was 1:4 and span 85 was added as the surfactant in accordance with the Box-Behnken design. The emulsion was centrifuged at 4000rpm for $1 \mathrm{~min}$ and thereafter excess cyclohexane was decanted. The remaining concentrated SMM emulsion was frozen at $-80^{\circ} \mathrm{C}$ for $12 \mathrm{hrs}$ before being lyophilized for $48 \mathrm{hrs}$.

\subsection{Determination of the stability of the optimized SMM emulsion}

The dispersion state of the W/O emulsion obtained in the formulation of SMMs was measured using a Turbiscan $\mathrm{Lab}^{\circledR}$ (Turbiscan $\mathrm{Lab}^{\mathrm{TM}}$, Formulaction SA, L'Union, France) which assessed the degree of reversible colloidal phenomena such as creaming and 
sedimentation as well as irreversible phenomena such as coalescence and flocculation (Celia et al., 2009). The Turbiscan Lab ${ }^{\circledR}$ Expert software was used to analyze transmitted (T) and backscattered (BS) light according to Eq. 1 and in reference to the Mie theory represented by Eq. 2.

$B S=1 / \sqrt{\lambda^{*}}$

$\lambda^{*}(d, \Phi)=2 d /\left[3 \Phi(1-g) Q_{s}\right]$

Where $\lambda^{*}$ is the photon transport mean free path, $\Phi$ is the volume fraction of the particles, $d$ is the particle mean diameter and $g$ (asymmetry factor) and $Q_{s}$ (scattering efficiency factor) are optical parameters given by the Mie theory. A disposable flat bottomed cylindrical glass cell was carefully filled to approximately $42 \mathrm{~mm}$ height with the optimized SM emulsion $(20 \mathrm{mLs})$ then placed into the Turbiscan ${ }^{\circledR}$ Lab instrument for synchronous dual measurement of light that transmitted thought and light that was back-scattered at a $45^{\circ}$ angle in reference to the incident light. Pulsed near infrared light $(\lambda 880 \mathrm{~nm})$ was emitted from the source and optical detectors (photodiodes) received the transmitted and the backscattered light. Measurements were performed at $25^{\circ} \mathrm{C}$ and the Turbiscan ${ }^{\circledR}$ Lab was configured to perform continuous scans, performing a scan every 6 minutes, over a 55mm cell length from bottom to top for an hour. Variation in particle volume fraction $(\Phi)$ on particle migration (sedimentation of creaming) and the mean particle diameter $(d)$ due to coalescence resulted in variation in the quantity of transmitted and backscattered light. The measured amount of transmitted and backscattered light was then interpreted and used to describe the dispersion state (stability) of the emulsion (Mengual et al., 1999; Lemarchand et al., 2003; Celia et al., 2009).

\subsection{Morphological characterization of the SMMs}

The shape and surface morphology of the SMMs were determined using a transmission electron microscope (TEM, JEOL S100 Transmission Electron Microscope, Tokyo, Japan) set at $40000 \mathrm{X}$ magnification. The particle size of the SMMs was determined by measuring particle diameter through a process of dynamic light scattering using a Zetasizer (Zetasizer Nano ZS, Malvern Instruments Ltd, Worcestershire, United Kingdom). Particle surface charge was evaluated by measuring the zeta potential of the SMMs using the Zetasizer (Garcia et al., 2011). SMM powder (5mg) samples were each dispersed in $30 \mathrm{mLs}$ of MilliQ water and passed through $0.22 \mu \mathrm{m}$ Minisart ${ }^{\circledR}$ non-pyrogenic single use filters (Sartorius Stedim Biotech, Goettingen, Germany) into quartz cuvettes and specialized zeta potential 
containers respectively which were then inserted into the Zetasizer to determine particle size and zeta potential (Garcia et al., 2011).

\subsection{Fourier transform infrared (ATR-FTIR) spectroscopy analysis}

ATR-FTIR spectra of native PEC, MUC, PEG, AZT, blank PEG-encl-PEC:MUC SMMs and the AZT-loaded PEG-encl-PEC:MUC SMMs were obtained using a Perkin Elmer Spectrum 2000 FTIR spectrometer fitted with a MIRTGS detector (PerkinElmer Spectrum 100, Llantrisant, Wales, UK). Dry powder samples were directly analyzed over a wave number range of $650-4000 \mathrm{~cm}^{-1}$ with a resolution of $4 \mathrm{~cm}^{-1}$ and 10 scans per spectrum were performed at a direct contact force of $120 \mathrm{~N}$ on a universal diamond ATR top-plate. Distinct absorption peaks were used to verify the constitution of the SMMs by comparing them to the peaks on the native MUC, PEC, PEG and AZT.

\subsection{Thermal analysis}

The thermal properties of PEC, MUC, AZT and the AZT-loaded SMMs were analyzed by DSC. The analysis was conducted on a differential scanning calorimeter (Mettler Toledo, DSC1, STARe System, Schwerzenback, Switzerland) which was calibrated for temperature and enthalpy using indium and zinc. All experiments were performed at a heating rate of $10^{\circ} \mathrm{C} \mathrm{min}^{-1}$ under a dry nitrogen atmosphere (Afrox, Germiston, Gauteng, South Africa) which flowed at a rate of $200 \mathrm{mLmin}^{-1}$ acting as the purge gas in order to reduce sample oxidation. Samples were placed in $40 \mu \mathrm{L}$ aluminium pans and heated from $-10^{\circ} \mathrm{C}$ to $110^{\circ} \mathrm{C}$ and kept at $110^{\circ} \mathrm{C}$ for 3 minutes. This was done to evaporate any moisture in the sample and to eliminate any thermal history. The samples were then quenched from $110^{\circ} \mathrm{C}$ to $-10^{\circ} \mathrm{C}$ at a rate of $20^{\circ} \mathrm{C} \mathrm{min}^{-1}$. The midpoint melting point $\left(T_{\mathrm{m}}\right)$ and heat of fusion $(\Delta H)$ which were used for characterization were obtained from the melting point depression of the peaks generated on the experimental DSC curves on heating the samples from -10 to $300^{\circ} \mathrm{C}$.

\subsection{Thermogravimetric analysis}

Thermal degradation analysis was performed on 10-20mg samples of PEC, MUC, AZT and AZT-loaded PEG-encl-PEC:MUC SMMs which were contained in ceramic pans under nitrogen atmosphere using a TGA 4000 thermogravimetric analyzer (PerkinElmer Inc., Massachusetts, USA). The experiments were run at $10^{\circ} \mathrm{Cmin}^{-1}$ from $50-500^{\circ} \mathrm{C}$. Thermograms obtained and their first derivatives revealed the thermal degradation properties of the samples. 


\subsection{X-ray diffraction analysis}

The crystalline and amorphous nature of the AZT-loaded SMMs and their individual compositional components was investigated using a Rigaku MiniFlex600 Benchtop X-ray Diffractometer (Rigaku Corporation, Tokyo, Japan) fitted with; a $600 \mathrm{~W}$ (40Kv-15mA) X-ray generator, a counter monochromator to cut X-rays other than $\mathrm{Cu} \mathrm{Ka} \mathrm{X-rays} \mathrm{and} \mathrm{a} \mathrm{high}$ intensity $\mathrm{D} /$ tex ultra-high speed $1 \mathrm{D}$ detector. Experimental temperature was maintained at $19^{\circ} \mathrm{C}$. The diffractometer was operated using the Rigaku MiniFlex Guidance software, version 1.2.0.0 and data was analyzed using the Rigaku PDXL Basis software able to perform integrated intensity calculations. Measurement parameters included a divergence slit (DS) of $1.25^{\circ}$, scattering slit (SS) of $1.25^{\circ}$, a $0.3 \mathrm{~mm}$ receiving slit (RS) and a goniometer radius of $150 \mathrm{~mm}$. Each powder sample of PEC, MUC, AZT and AZT-loaded PEG-enc/PEC:MUC SMMs was pressed flat onto a square grooved glass slide sample holder. Measurements were performed by scanning each sample at $0.01-100 \% / \mathrm{min}$ over a diffraction angle range of $3^{\circ}-60^{\circ} 2 \theta$. The XRD diffractograms generated were used to evaluate the crystallinity of the respective samples (Santos et al., 2003; Mishra et al., 2008; Kumar et al., 2010; Raviolo and Briñón, 2011).

\subsection{Drug encapsulation and release from SMMs}

The percentage encapsulation efficiency (EE) of the optimized SMMs was determined according to Eq. 3 (Garcia et al., 2011).

$\mathrm{EE}(\%)=$ Actual amount of AZT in SMMs/theoretical amount of AZT $\times 100$

Where, the actual amount of AZT in SMMs was measured on a $100 \mathrm{mg}$ sample. SMMs (100mg) were added to $100 \mathrm{~mL}$ of MilliQ water, ultrasonicated for 10 minutes, vortexed for 5 minutes and then allowed to release the remaining AZT form the SMMs over 48 hours whilst in an orbital shaker bath that rotated at $20 \mathrm{rpm}$ and was set at $37^{\circ} \mathrm{C}$. The amount of $\mathrm{AZT}$ that was encapsulated in the SMMs was then computed form the UV absorbance values measured using a nanophotometer (NanoPhotometer ${ }^{\mathrm{TM}}$, Implen $\mathrm{GmbH}$, Munchen, Germany) at ambient temperature $\left(25^{\circ} \mathrm{C}\right)$ and at a UV absorbance lambda max of $267 \mathrm{~nm}$. Determination of EE was performed in triplicate. The theoretical amount of AZT was the proportional amount of AZT in the 100mg of SMMs in reference to the loading dose. AZT release from PEG-encl-PEC:MUC SMMs was performed using the dialysis membrane technique over 24 hours in a 100mL container filled with SVF (Gupta et al., 2011; Woolfson et al., 2010; Shaikh et al., 2009). The dissolution media (simulated vaginal fluid, SVF), was prepared according to Owen and Katz's as presented in Table 3 (Owen and Katz, 1999). 
The drug-loading capacity was computed accordingly based on the various drug:polymer ratios.

\begin{tabular}{ll}
\hline Table 3: Vaginal fluid stimulant (VFS) 1L (Owen and Katz, & Quantity (g) \\
1999)VFS Component & 3.510 \\
\hline Sodium chloride $(\mathrm{NaCl})$ & 1.400 \\
Potassium hydroxide $(\mathrm{KOH})$ & 0.222 \\
Calcium hydroxide $\mathrm{Ca}(\mathrm{OH})_{2}$ & 0.018 \\
Bovine serum albumin $(\mathrm{BSA})$ & 2.000 \\
Lactic acid & 1.000 \\
Acetic acid & 0.160 \\
Glycerol & 0.400 \\
Urea & 5.000 \\
Glucose & \\
\hline
\end{tabular}

Drug release was also performed in phosphate buffered saline (PBS pH 7.4) which acted as simulated semen (Gupta et al., 2011; Woolfson et al., 2010; Owen and Katz, 2005). The AZT-loaded SMMs and $2 \mathrm{mLS}$ SVF or PBS (similar medium as the bulk $100 \mathrm{~mL}$ dissolution medium) were added into a dialysis tubing which had a molecular weight cut off of $12 \mathrm{kDa}$ obtained from Sigma-Aldrich Inc., St. Louis, USA) (Gupta et al., 2011; Shaikh et al., 2009). The tubing was securely tied at both ends and then suspended in a dissolution media filled $100 \mathrm{~mL}$ container that was placed in an orbital shaker incubator (Orbital Shaker Incubator, LM-530D, Yihder Technology CO., LTD, Jhonghe City, Taipei County, Taiwan, Republic of China) which was set at $37^{\circ} \mathrm{C}$ and rotated at 20rpm (Woolfson et al., 2010). Dissolution media aliquots $(200 \mu \mathrm{L})$ were withdrawn at predetermined time intervals and Ultraviolet (UV) quantification of the amount of drug released was performed using the nanophotometer. Dissolution tests were performed in triplicate. Dissolution profiles were generated from the amounts of AZT released over 24 hours and these profiles were used to characterize in vitro AZT release kinetics from the SMMs. The ability of the macromolecular SMMs to modulate AZT release was evaluated by determining the mean dissolution time (MDT) according to Eq. 4 and the dissolution efficiency (DE) which is the area under the dissolution curve up to a certain time, $t$, expressed as a percentage of the rectangular area described by $100 \%$ dissolution in the same time, according to Eq. 5 (Khan, 1975; Costa and Lobo, 2001, Garcia et al., 2011).

$M D T=\frac{\sum_{i=1}^{n} t_{i} M_{t}}{M_{\infty}}$

Where $M_{t}$ is the fractional dose released in time $t_{i}=\left(t_{i}+t_{i-1}\right) / 2$ and $M_{\infty}$ corresponds to the loading dose 
$D E=\frac{\int_{0}^{t} y \times d t}{y_{100} \times t} \times 100 \%$

Where $\mathrm{y}$ is the percentage of drug dissolved at time t. The $\mathrm{pH}$ responsiveness of the SMMs was tested by comparing the dissolution efficiencies, MDTs and computing the similarity factor $\left(f_{2}\right)$ and the difference factor $\left(f_{1}\right)$ of the dissolution profiles obtained in SVF and in PBS according to Eqs. 6 and 7 (Moore and Flanner, 1996; O'Hara et al., 1998; Costa et al., 2001; Zhang et al., 2010).

$$
\begin{aligned}
f_{2} & =50 \log \left\{\left[1+1 / n \sum_{t=1}^{n} W_{t}\left(R_{t}-T_{t}\right)^{2}\right]^{-0.5} \times 100\right\} \\
f_{1} & =\frac{\sum_{t=1}^{n}\left|R_{t}-T_{t}\right|}{\sum_{t=1}^{n} R_{t}} \times 100 \%
\end{aligned}
$$

Where $\mathrm{n}$ is the number of sampling points, $W_{t}$ is an optional weight factor, $R_{t}$ is the reference assay at time $t$ and $T_{t}$ is the test assay at time $t$. The dissolution profile of the PEG-enclPEC:MUC SMMs in SVF and in PBS was fitted into 14 different kinetic models. The best fit parameters and the adjusted coefficient of determination $\left(R^{2}\right.$ adjusted $)$, which is regarded as the goodness of fit parameter, was obtained. $\mathrm{R}^{2}$ adjusted was viewed as a measure of how close the dissolution profile of the SMMs fitted into the chosen kinetic model, with values of $\mathrm{R}^{2}$ adjusted that increasingly approached one showing the closest model fit as opposed to values smaller than 1 (Zhang et al., 2010).

\section{Results and Discussion}

\subsection{Preparation and optimization of the macromolecular SMMs}

All 15 formulations from the design template produced SMMs of varying particle size, zeta potential and had different MDTs (Table 4). The obtained results were entered into the MINITAB ${ }^{\circledR}$ software to yield four possible optimized formulations and the most appropriate optimized formulation had a composite desirability (D) of 0.865 , PS desirability ( $d_{P S}$ ) of 1.000 , ZP desirability $\left(\mathrm{d}_{\mathrm{ZP}}\right)$ of 0.825 and MDT desirability $\left(\mathrm{d}_{\mathrm{MDT}}\right)$ of 0.785 . Independent and dependent parameters that had a desirability of 1,000 were regarded as the most optimum and those that had a desirability of 0.000 were the least optimum parameters. The optimal formulation had independent parameters; ST of 6.28 minutes, SC of $1.64(\% \mathrm{v} / \mathrm{v})$ and a D:P ratio of 1:1 which gave predicted SMM responses of; PS $215.19 \mathrm{~nm}$, ZP $-39.13 \mathrm{mV}$ and a MDT of 5.52 hours. Since the encapsulation efficiency ( $\approx 90 \%)$ and percentage drug loading showed no significant difference among the formulations, these two parameters were not included as responses during formulation optimization. Linear regression of independent 
parameters and responses using MINITAB $^{\circledR}$ generated relationships described by the polynomial Eqs. 8, 9, and 10 (Karnachi and Khan, 1996). Where the coefficient of determination of PS $\left(R^{2}{ }_{P S}\right)$ was $51.4 \%$, ZP $\left(R^{2}{ }_{Z P}\right)$ was $90.1 \%$ and that of MDT $\left(R^{2}{ }_{M D T}\right)$ was 85.6\% (Karnachi and Khan, 1996).

$P S=-1719.600+163.070[S T]+1247.470[S C]+637.950[D: P]-3.900[S T]^{2}-158.33[S C]^{2}-$ 127.530[D:P] $\left.{ }^{2-66.800[S T *}{ }^{\star} S C\right]+24.600\left[S T^{\star} D: P\right]-227.200\left[S C{ }^{*} D: P\right]$

$Z P=40.125-4.925[S T]+13.250[S C]-189.100[D: P]+0.580[S C]^{2}-20.400[S C]^{2}-26.400[D: P]^{2}-$ $4.160\left[S T^{*} S C\right]+3.000\left[S T^{*} D: P\right]+122.800[S C * D: P]$

MDT $=-34.951+3.267[S T]+41.139[S C]-20.998[D: P]-0.093[S T]^{2}-11.896[S C]^{2}-4.624[D: P]^{2}-$ $0.921\left[S T^{*} S C\right]-0.415\left[S T^{*} D: P\right]+10.104[S C * D: P]$

The correlation of the independent parameters; ST, SC and D:P ratio to the responses; PS, ZP and MDT was further illustrated as response mesh plots presented in Fig. 1 (Karnachi and Khan, 1996).

Table 4: Responses obtained from in vitro testing of the 15 design formulation's independent parameters

\begin{tabular}{|c|c|c|c|c|c|}
\hline $\begin{array}{l}\text { Formulation } \\
\text { number }\end{array}$ & $\begin{array}{c}\text { Particle size } \\
(\mathrm{nm})\end{array}$ & $\begin{array}{c}\text { Zeta potential } \\
(\mathrm{mV})\end{array}$ & $\begin{array}{l}\text { Encapsulation } \\
\text { efficiency (\%) }\end{array}$ & $\begin{array}{c}\text { Drug loading } \\
(\%)\end{array}$ & MDT (hours) \\
\hline 1 & $186.1 \pm 4.267$ & $-42.7 \pm 2.350$ & $93.82 \pm 4.03$ & $31.27 \pm 1.26$ & 4.852 \\
\hline 2 & $311.0 \pm 13.360$ & $-35.4 \pm 0.503$ & $91.21 \pm 4.73$ & $39.08 \pm 2.78$ & 4.825 \\
\hline 3 & $143.6 \pm 21.110$ & $-33.7 \pm 1.460$ & $90.19 \pm 2.83$ & $38.64 \pm 2.45$ & 3.572 \\
\hline 4 & $229.1 \pm 52.030$ & $-40.2 \pm 1.400$ & $87.22 \pm 1.97$ & $37.38 \pm 0.28$ & 3.845 \\
\hline 5 & $228.7 \pm 12.510$ & $-28.1 \pm 0.894$ & $94.31 \pm 3.17$ & $40.41 \pm 2.13$ & 4.895 \\
\hline 6 & $220.0 \pm 4.768$ & $-29.7 \pm 0.144$ & $90.62 \pm 4.43$ & $45.31 \pm 1.39$ & 5.612 \\
\hline 7 & $223.2 \pm 12.390$ & $-30.5 \pm 0.193$ & $92.72 \pm 2.03$ & $46.36 \pm 1.81$ & 6.300 \\
\hline 8 & $262.9 \pm 3.964$ & $-33.1 \pm 0.535$ & $88.36 \pm 2.09$ & $37.88 \pm 2.42$ & 5.339 \\
\hline 9 & $203.1 \pm 2.989$ & $-41.6 \pm 0.671$ & $91.88 \pm 3.04$ & $39.37 \pm 2.31$ & 5.952 \\
\hline 10 & $231.3 \pm 4.634$ & $-32.4 \pm 0.618$ & $90.12 \pm 1.32$ & $45.06 \pm 2.25$ & 5.209 \\
\hline 11 & $220.6 \pm 6.202$ & $-34.2 \pm 0.948$ & $86.99 \pm 1.67$ & $28.99 \pm 0.77$ & 5.958 \\
\hline 12 & $239.5 \pm 9.255$ & $-33.3 \pm 0.415$ & $93.28 \pm 4.74$ & $31.09 \pm 1.56$ & 4.906 \\
\hline 13 & $228.1 \pm 5.899$ & $-47.4 \pm 0.536$ & $92.32 \pm 3.57$ & $46.16 \pm 1.16$ & 4.349 \\
\hline 14 & $269.3 \pm 13.450$ & $-47.2 \pm 0.860$ & $91.55 \pm 3.44$ & $30.51 \pm 2.28$ & 4.695 \\
\hline 15 & $321.1 \pm 17.370$ & $-35.4 \pm 0.501$ & $90.48 \pm 2.21$ & $38.77 \pm 2.17$ & 5.533 \\
\hline
\end{tabular}



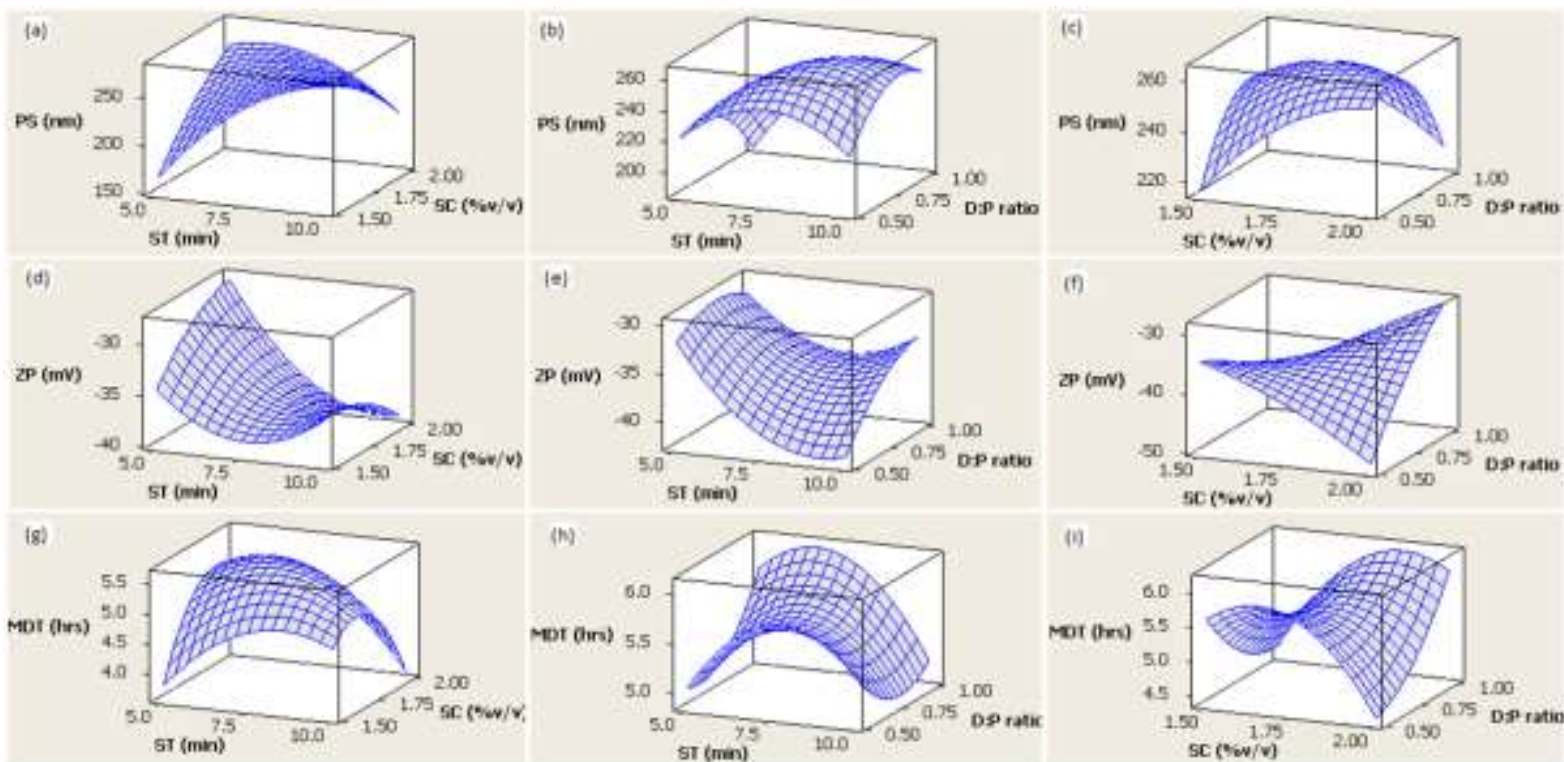

Fig. 1. Response mesh plots correlating dependent formulation parameters/ responses; PS, ZP and MDT to their independent parameters; ST, SC and D:P ratio.

\subsection{Sub-microsphere emulsion stability}

The delta transmission and delta back scattering profiles of the optimised SMM emulsion depicted in Fig. 2 shows that the SMM emulsion was generally stable with some local destabilization (sedimentation) as observed by the gradual decrease in the delta backscattering (DeltaBS) signal at the top end of the glass cell. The middle and the left of the profile was relatively unchanged which meant that the emulsion was stable over the hour long test duration and no global destabilization (coalescence) and no creaming occurred to the SMM emulsion during the total experimentation duration (Fig. 2).

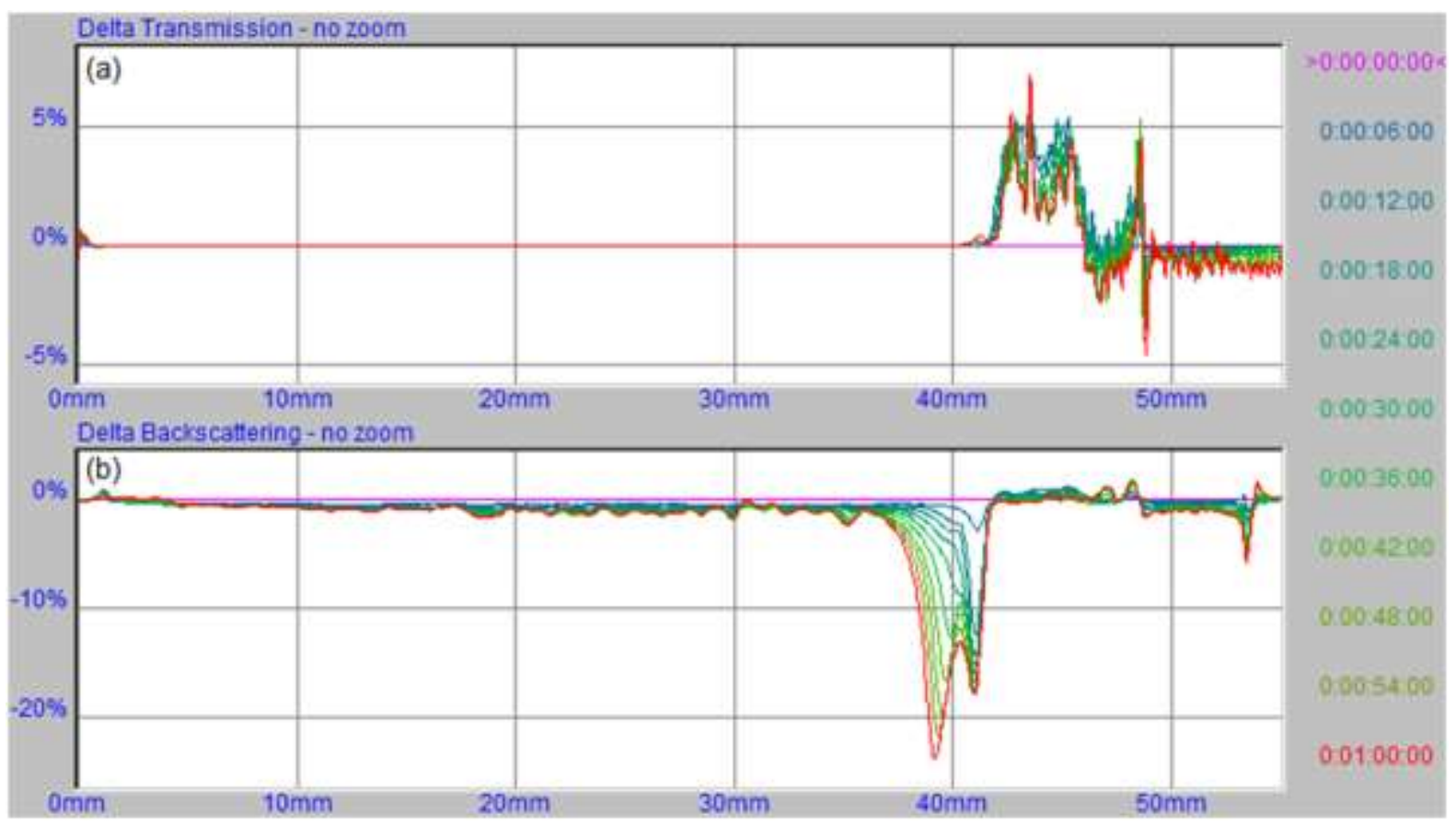

Fig. 2. Delta transmission and delta backscattering profiles of an optimized SMM emulsion obtained through continuous scans every 6 minute for 1 hour. 
During the SMM synthesis the emulsion is centrifuged within an hour followed by freezing at -80 degrees for 24 hours then lyophilized for 48 hours, therefore the minimum sedimentation that occurs as observed in Fig. 2 does not significantly affect the AZT-loaded PEG-enclPEC:MUC SMM formulation.

\subsection{Morphological characterization of AZT-loaded PEG-encl-PEC:MUC SMMs}

Well defined spherical SMMs were observed under TEM (Fig. 3). The mean particle size of the SMMs prepared from the 15 design formulations ranged from $143.6 \pm 21.110 \mathrm{~nm}, \mathrm{~F} 3$, to 321.1 $\pm 17.370 \mathrm{~nm}, \mathrm{~F} 15$, (Table 4). The optimized formulation had a mean particle size of $270.6 \pm 5.53 \mathrm{~nm}$ and had a stable polydispersity index (PDI) of $0.303 \pm 0.028$ as depicted in Fig. 4a. Zeta potential for the 15 design formulations ranged from $-28.1 \pm 0.894 \mathrm{mV}$ to $47.4 \pm 0.536 \mathrm{mV}$ (Table 4 ). The optimized formulation had a zeta potential of $-34.4 \pm 0.539 \mathrm{mV}$ signifying that the SMMs were not agglomerated (Fig. 4b).

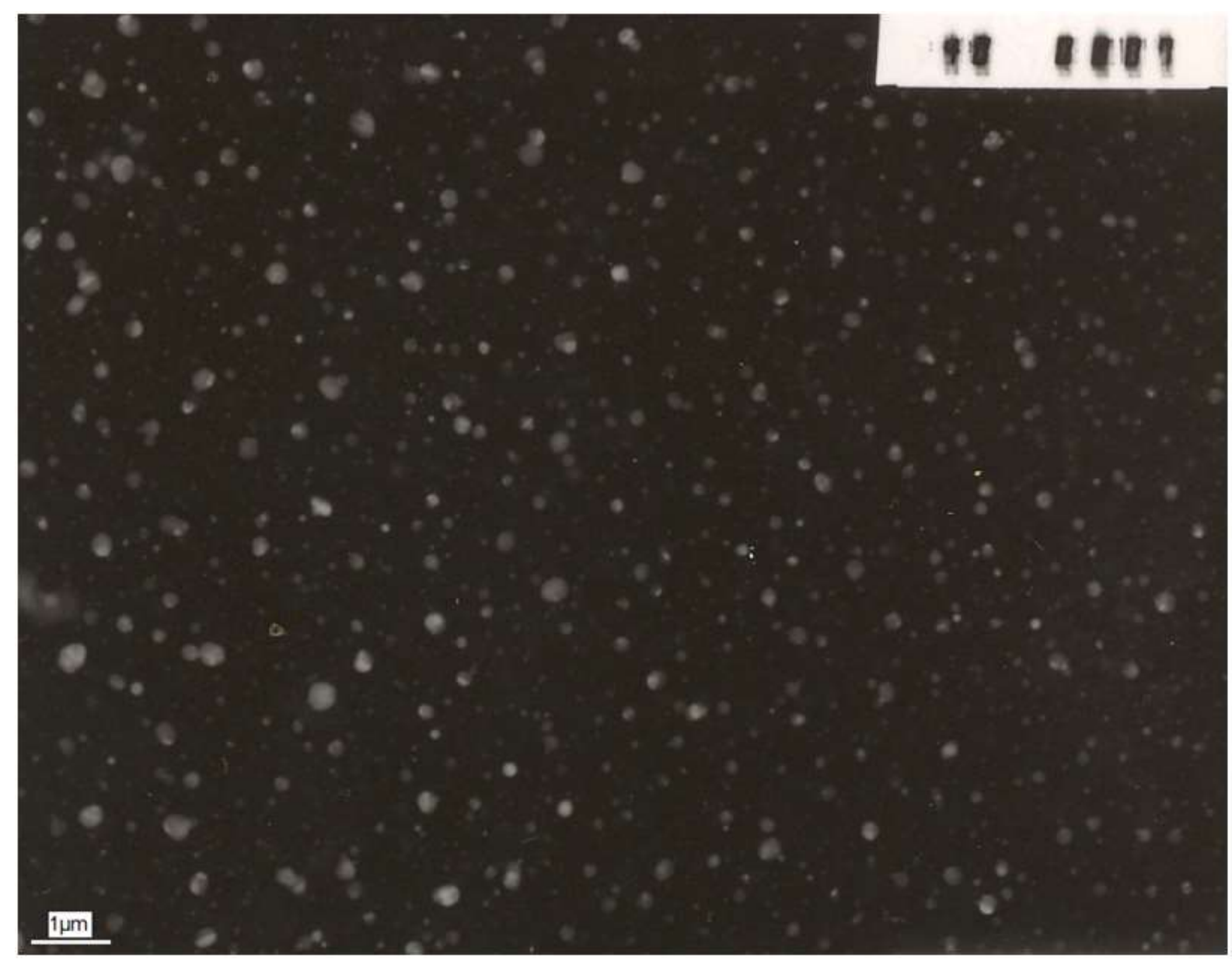

Fig. 3. Transmission electron micrograph of AZT-loaded PEG-encl-PEC:MUC SMMs at 40 000X magnification observed using a JEOL S100 Transmission Electron microscope. 

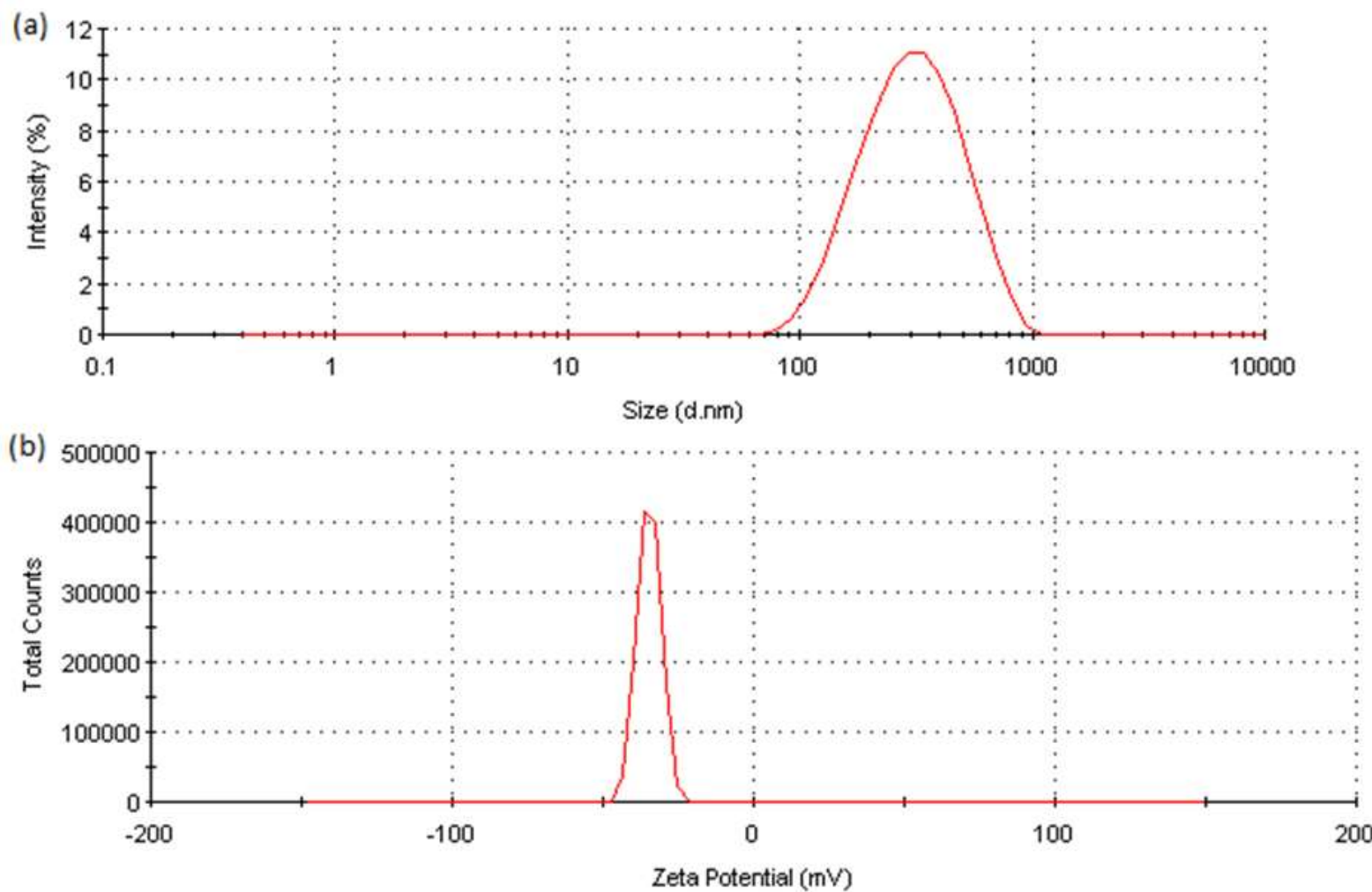

Fig. 4. (a) Particle size distribution with intensity and (b) zeta potential distribution graphs of the optimized SMMs obtained using a Zetasizer.

\subsection{ATR-FTIR analysis of components and the AZT-loaded PEG-encl-PEC:MUC SMMs}

FTIR spectrum of PEG-encl-PEC:MUC SMMs (Fig. 5) revealed a broad absorption peak extending from approximately $3000-3600 \mathrm{~cm}^{-1}$ due to the overlapped stretching vibrations of hydroxyl $(-\mathrm{OH})$ groups present in MUC, PEC, AZT and PEG and the carboxyl (-COOH) groups of PEC and MUC (Kumar et al., 2010; Mashingaidze et al., 2013). The broadening of this peak as compared to those in PEC (Fig. 5c), MUC (Fig. 5b), PEG (Fig. 5e) and AZT (Fig. $5 \mathrm{~d}$ ) was due to the participation of the $-\mathrm{OH}$ and $\mathrm{COOH}$ groups in $\mathrm{H}$-bonding. The SMM absorption peaks observed at $2933 \mathrm{~cm}^{-1}$ and $2854 \mathrm{~cm}^{-1}$ were attributed to strong stretching vibrations of the alkane groups; $-\mathrm{CH}_{3},-\mathrm{CH}_{2}$, and $-\mathrm{CH}$ that are found in PEC (peak $2935 \mathrm{~cm}^{-}$ ${ }^{1}$ ), MUC (peak $2917 \mathrm{~cm}^{-1}$ ), and PEG $400\left(\right.$ peak $2867 \mathrm{~cm}^{-1}$ ).

AZT encapsulation in PEG-encl-PEC:MUC SMM was confirmed by the presence of the absorption peak at $2105 \mathrm{~cm}^{-1}$ which was assigned to the stretching vibrations of the azide ($N_{3}$ ) group of AZT (Fig. 5a). The reduction in intensity of this peak as compared to those observed on the AZT ATR-FTIR spectrum (Fig. 5d) resulted from shielding of AZT as it was encapsulated within the PEG-encl-PEC:MUC SMMs and due to the participation of the $-\mathrm{N}_{3}$ group in $\mathrm{H}$-bonding with $-\mathrm{OH}$ and $-\mathrm{COOH}$ groups found in $\mathrm{PEC}, \mathrm{MUC}$ and $\mathrm{PEG}$. The peak that was observed at $1742 \mathrm{~cm}^{-1}$ on the AZT-loaded PEG-encl-PEC:MUC SMM ATR-FTIR spectra (Fig. 5a) resulted from the contribution made by strong stretching vibrations of the 
ester bond present in PEC which was approximately 55-65\% esterified (GENU ${ }^{\circledR}$ pectin USP/100, CP Kelco ApS, Lille Skensved, Denmark). A strong amide I (-C=O) peak (1682cm $\left.{ }^{1}\right)$ was observed on the SMM spectrum that contributed to the strong stretching vibrations of the amide I bond in MUC (peak $1636 \mathrm{~cm}^{-1}$ ) and AZT, peak $1641 \mathrm{~cm}^{-1}$ and $1670 \mathrm{~cm}^{-1}$ (Patel et al., 2003; Tam et al., 2005). The ATR-FTIR spectra of SMMs, PEC, MUC, PEG and AZT depicted several peaks in the finger-print region $(1450-650 \mathrm{~cm})$ which were consistent with what is reported in literature and expected for the AZT-loaded SMMs (Mishra et al., 2008; Patel et al 2003; Ravi et al., 2008). No apparent differences were observed between the FTIR spectra of drug-loaded and drug-free PEG-encl-PEC:MUC SMMs except for the presence of vibrational peaks corresponding to AZT in the former.

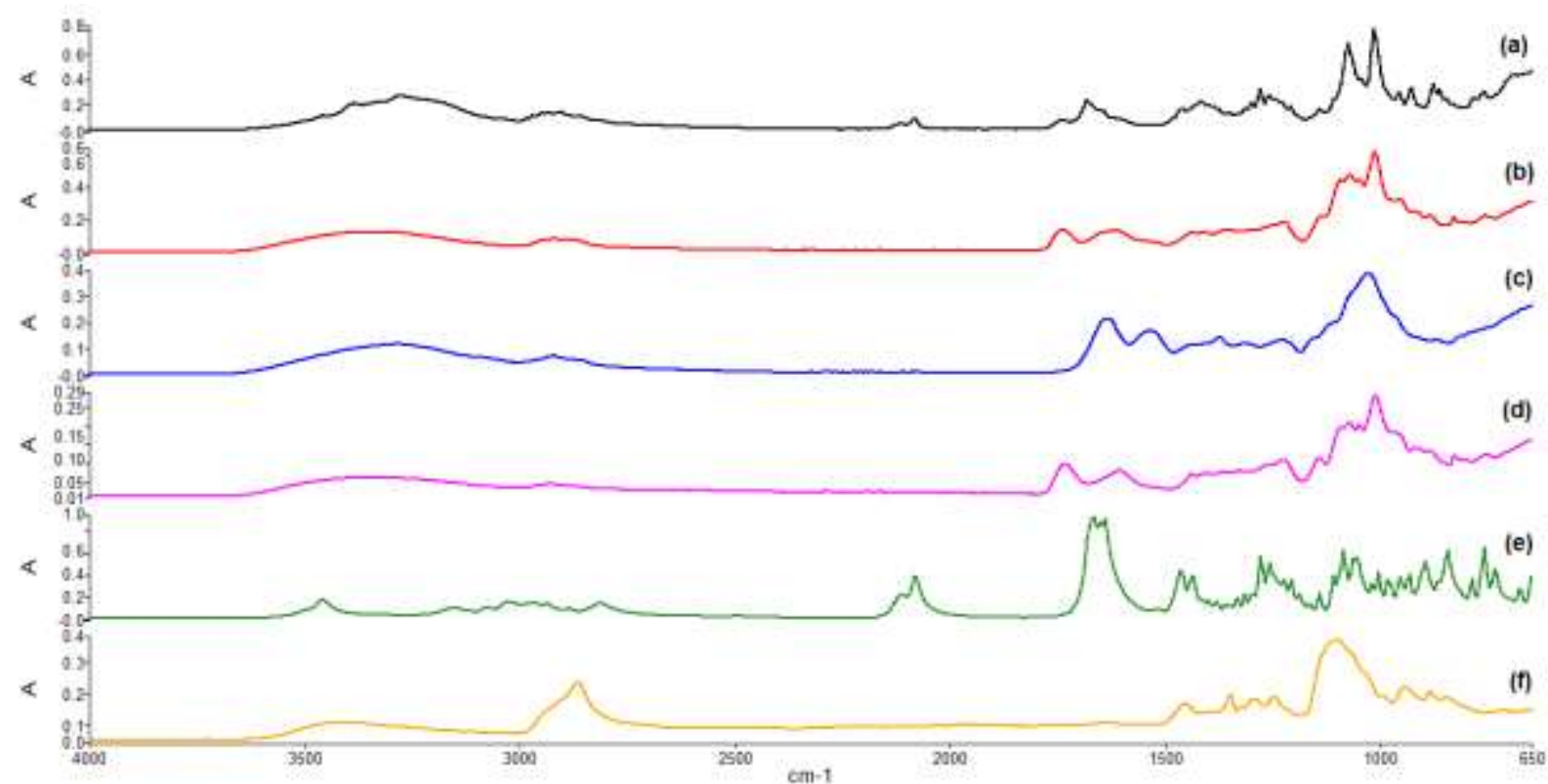

Fig. 5. ATR-FTIR spectra of; (a) AZT-loaded PEG-encl-PEC:MUC SMMs, (b) blank PEG-enc/-PEC:MUC SMMs, (c) pristine MUC, (d) pristine PEC, (e) pristine AZT, and (f) pristine PEG.

\subsection{Assessment of differential scanning calorimetry profiles}

DSC thermograms of AZT-loaded PEG-encl-PEC:MUC SMMs, MUC, PEC and AZT are depicted in Fig. 6. AZT-loaded PEG-encl-PEC:MUC SMMs had a broad endothermic peak with melting point peak maxima at $113.67^{\circ} \mathrm{C}$ and a measured heat of fusion of $9.75 \mathrm{Jg}^{-1}$ as well as an exothermic crystallization peak at $230.81^{\circ} \mathrm{C}$ (Fig. 6a). SMM components; MUC had an melting point represented by an endothermic peak maxima at $101.36^{\circ} \mathrm{C}$ (Fig. $6 \mathrm{~b}$ ), PEC had a melting endothermic peak at $120.73^{\circ} \mathrm{C}$ as well as an exothermic crystallization peak at $236.17^{\circ} \mathrm{C}$ (Fig. $6 \mathrm{C}$ ) and the model drug, AZT, had an onset melting point at $122.88^{\circ} \mathrm{C}$ and an exothermic crystallization peak at $240.34^{\circ} \mathrm{C}$ (Fig. 6d) that were consistent with results reported in literature (Araújo et al., 2003). The thermal DSC thermogram of AZT-loaded 
PEG-encl-PEC:MUC SMMs confirms SMM composition and was a result of the thermal contribution of PEC, MUC, PEG and AZT.

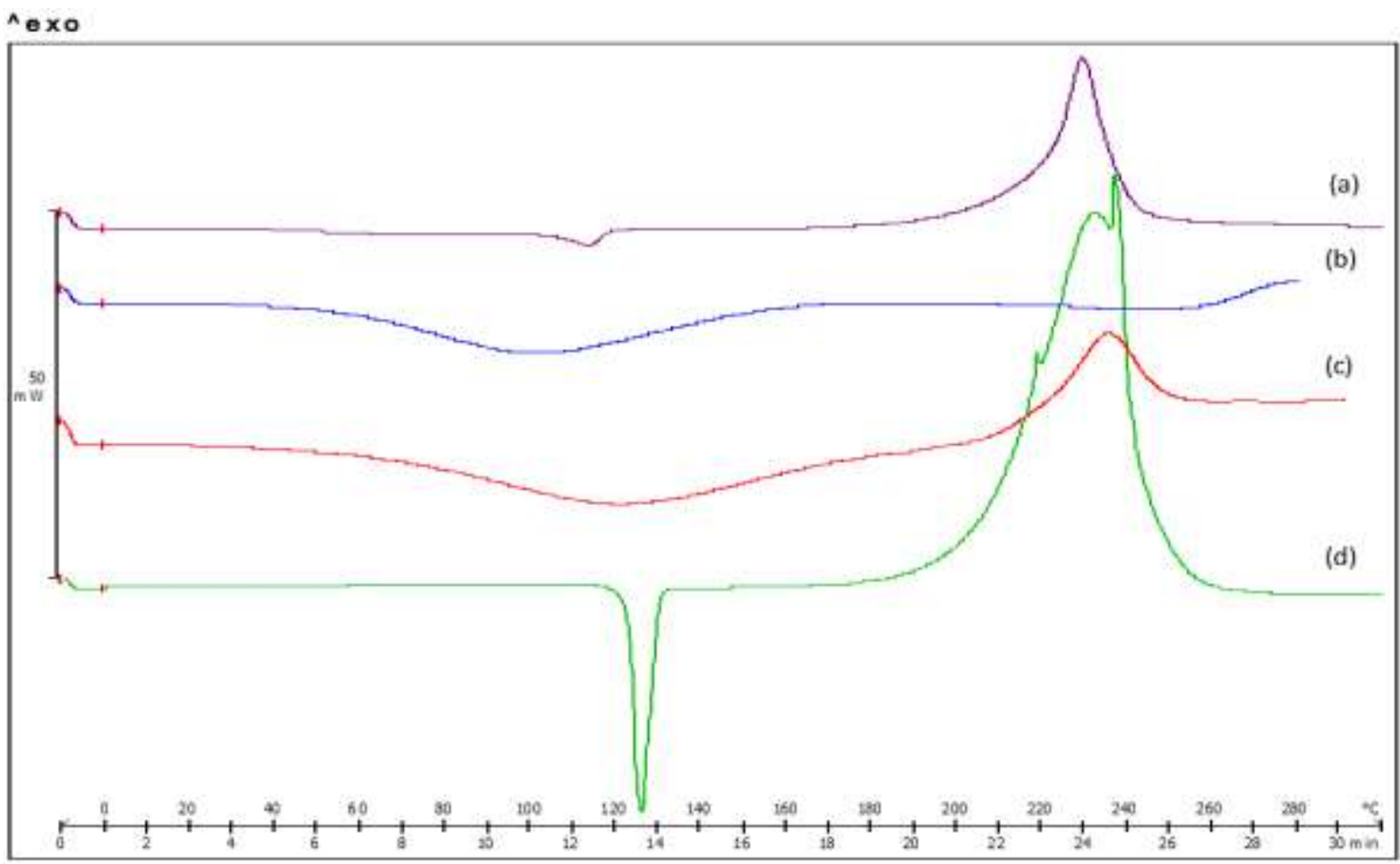

Fig. 6. DSC thermograms of (a) AZT-loaded PEG-enc/-PEC:MUC SMMs, (b) pristine MUC, (c) pristine PEC, (d) pristine AZT.

\subsection{Thermogravimetric analysis of AZT loaded SMMs and their native constituents}

The thermal degradation of AZT-loaded PEG-encl-PEC:MUC SMMs as measured by TGA produced the thermogram depicted in Fig. 7a (solid). The SMM TGA thermogram depicts indistinctive one-step degradation with weight loss of approximately $84 \%$ on heating from 50 $500^{\circ} \mathrm{C}$. However, its first derivative TGA (DTGA, dotted) revealed that the SMMs degraded in three major steps as revealed by DTGA peaks at $239.60^{\circ} \mathrm{C}, 287.90^{\circ} \mathrm{C}$ and $409.77^{\circ} \mathrm{C}$ that correspond to the points of inflection on the SMM TGA thermogram. It is vital to compare the SMM TGA thermogram with that of all the components from which the SMMs were formulated i.e. PEC, MUC, PEG and AZT as presented in Fig. 7b. Fig. 7c shows the TGA (solid) and DTGA (dotted) thermograms of PEC. Thermal degradation of PEC was shown to take place in two steps; the first was a minor step that took place from $50-150^{\circ} \mathrm{C}$ resulting in $7.919 \%$ weight loss and a point of inflection at $95.03^{\circ} \mathrm{C}$ which may have been a result of dehydration. The second and major PEC degradation step had an extrapolated onset and end at $233.54^{\circ} \mathrm{C}$ and $276.92^{\circ} \mathrm{C}$ respectively with its point of maximum degradation represented by the DTGA (peak $246.01^{\circ} \mathrm{C}$ ). This second degradation step resulted in a weight loss of approximately $59 \%$ and was most likely due to the depolymerization of PEC carbohydrate chains (Kumar et al., 2010). PEC weight loss of approximately $66.98 \%$ was 
experienced from $50-500^{\circ} \mathrm{C}$. The first DTGA peaks of the SMMs, $239.60^{\circ} \mathrm{C}$ (Fig. 7a) and PEC, $246.01^{\circ} \mathrm{C}$ (Fig. 7c) are observed to be close; therefore, PEC degradation might have contributed to the initial degradation of the SMMs (Fig. 7a).

Thermal degradation of MUC was also observed to occur in two steps, with the first minor step occurring from $50-150^{\circ} \mathrm{C}$ which resulted in a corresponding DTGA peak at $90.63^{\circ} \mathrm{C}$ that resulted in weight loss of approximately $9.14 \%$ (Fig. $7 d$ ). This step was likely a result of the evaporation of bound water molecules from the MUC macromolecules. This first step was then followed by a major degradation step which had an extrapolated onset and end at $264.58^{\circ} \mathrm{C}$ and $330.64^{\circ} \mathrm{C}$ respectively with its point of inflection corresponding to the DTGA peak at $286.50^{\circ} \mathrm{C}$. This major degradation step was possibly a result of the depolymerization of oligosaccharide side-chains in MUC. Overall, there was a total weight loss of $66.17 \%$ on heating MUC from $50-500^{\circ} \mathrm{C}$. Due to the closeness of the DTGA peaks of the corresponding second degradation steps of MUC, peak at $286.50^{\circ} \mathrm{C}$ (Fig. 7d) and SMMs, peak at $287.90^{\circ} \mathrm{C}$ (Fig. 7a), MUC was likely a contributor to the second degradation step of the AZT-loaded PEG-encl-PEC:MUC SMMs (Fig. 7a).

The model drug, AZT, thermally degraded in two major steps depicted by the DTGA peaks at $244.10^{\circ} \mathrm{C}$ (first step) and $310.70^{\circ} \mathrm{C}$ (second step) which might have contributed to the first and second SMM degradation steps (Figs. 7a and 7e). Degradation of AZT started at the extrapolated onset of $230.14^{\circ} \mathrm{C}$ then the second degradation step began before the first degradation step ended. The extrapolated end of the degradation of AZT was at $331.48^{\circ} \mathrm{C}$ and the total AZT weight loss on heating from $50-500^{\circ} \mathrm{C}$ was $77.935 \%$. The third degradation step of the SMMs, DTGA peak $409.77^{\circ} \mathrm{C}$ (Fig. 7a) was likely due to the degradation of PEG which degraded almost completely $(99.084 \%)$ in one major step with an extrapolated onset and end of $338.89^{\circ} \mathrm{C}$ and $393.97^{\circ} \mathrm{C}$ respectively (Fig. $7 \mathrm{f}$ ). The point of maximum degradation of PEG corresponds to the DTGA peak at $395.15^{\circ} \mathrm{C}$ (Fig. $\left.7 \mathrm{f}\right)$.

All the three degradation steps of the SMMs were shifted to the right as compared to the corresponding degradation steps of the respective components of the SMMs (PEC, MUC, AZT and PEG) signifying SMM thermal stability. The SMM thermal stability might have been brought about by the macromolecular ionic crosslinking using $\mathrm{Ca}^{2+}$ done in formulation of the SMMs as well as the intermolecular interactions possibly due to hydrogen bonding of the carboxyl, hydroxyl, amide, amine and azide groups of the SMM components. 

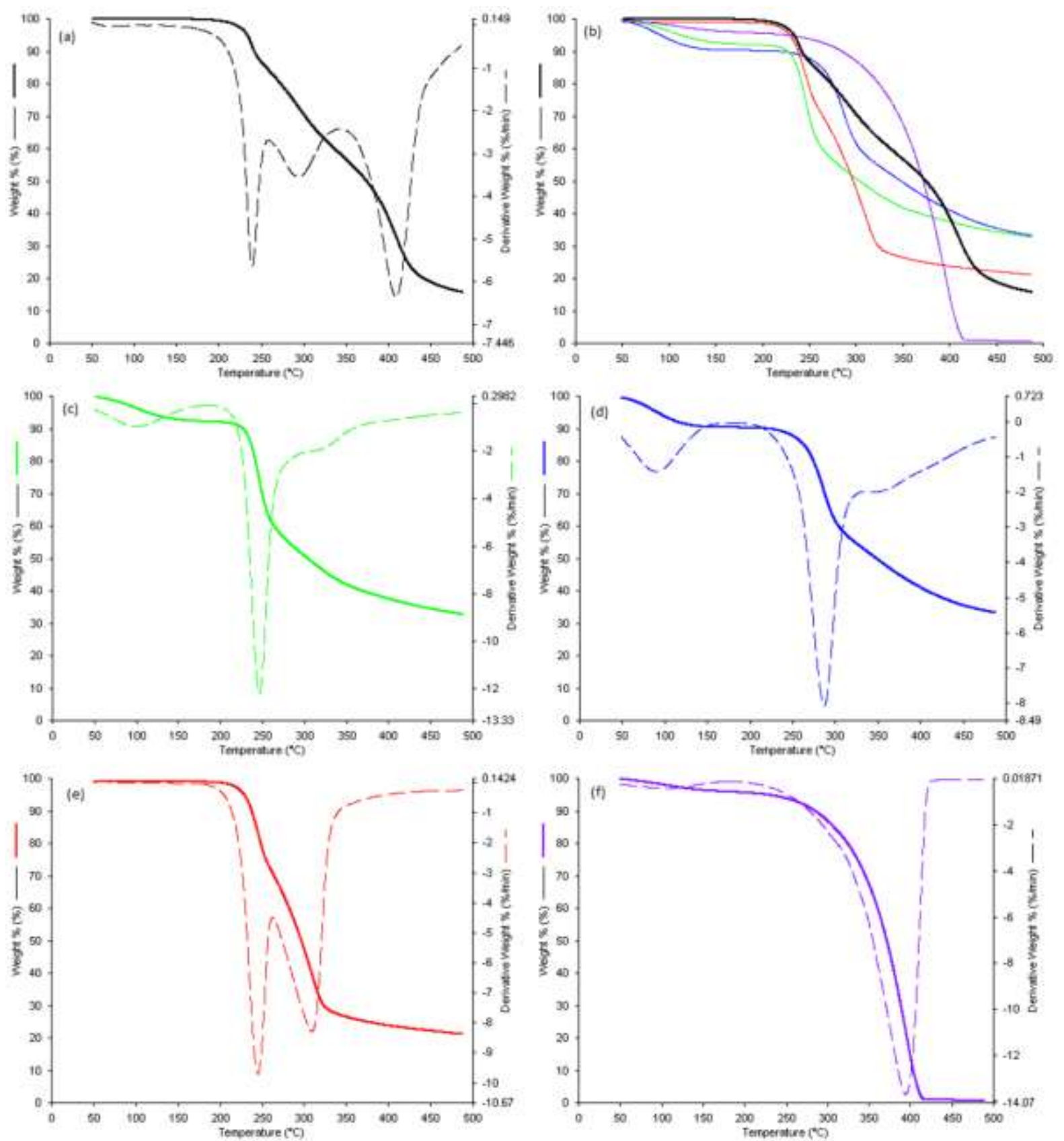

Fig. 7. The TGA (solid) and DTGA (dotted) thermograms of: (a) AZT-loaded PEG-encl-PEC:MUC SMMs; (b) (i) pristine MUC, (ii) pristine PEC, (iii) pristine AZT, (iv) PEG-encl-PEC:MUC+AZT MS and (v) pristine PEG; (c) pristine PEC; (d) pristine MUC; (e) pristine AZT and (f) pristine PEG.

\subsection{Evaluation of $x$-ray diffraction patterns}

Fig. 8 depicts the XRD diffractograms of; AZT-loaded PEG-encl-PEC:MUC SMMs, PEC, MUC and that of AZT. The diffractogram of AZT-loaded PEG-encl-PEC:MUC SMMs shows several sharp peaks that include major peaks at $2 \theta$ equal to $8.725^{\circ}, 14.535^{\circ}, 15.374^{\circ}$, $15.726^{\circ}, 16.987^{\circ}, 21.265^{\circ}, 22.093^{\circ}, 24.064^{\circ}, 26.825^{\circ}, 27.677^{\circ}, 29.181^{\circ}$ and $29.181^{\circ}$ which signify the crystalline nature of the AZT-loaded PEG-encl-PEC:MUC SMMs (Fig. 8a). The diffractogram of PEC depicts a broad peak at $2 \theta$ equal to $21.274^{\circ}$ indicating the amorphous nature of PEC (Fig. 8c) and the difractogram of MUC depicts a broad peak at $2 \theta$ equal to 
21.525 illustrating also the amorphous nature of MUC (Fig. 8b). AZT was shown to be crystalline by several sharp peaks that include major peaks at $2 \theta$ equal to $9.000^{\circ}, 14.891$, $15.725^{\circ}, 16.040^{\circ}, 17.304^{\circ}, 21.41^{\circ}, 21.640^{\circ}, 24.513^{\circ}, 27.140^{\circ}, 28.050^{\circ}$ and $29.500^{\circ}$ observed on its diffractogram shown in Fig. $8 \mathrm{~d}$ which are similar to the ones previously reported by Raviolo and Briñón (Raviolo and Briñón, 2011). The observed patten in the diffractograms in Fig. 8 shows that the crystallinity of AZT-loaded PEG-encl-PEC:MUC SMMs was a result of the contribution by the highly crystalline AZT. The peaks observed in the diffractogram of AZT-loaded PEG-encl-PEC:MUC SMMs were sharp as those observed for AZT but were shifted slightly to the left by less than $1^{\circ}$ and peak intensities were lower than those of observed for AZT which was probably because of the formation of a less crystaline complex when AZT was ecapsulated in the amorphous MUC and PEC based SMMs (Mishra et al., 2008; Santos et al., 2003, Shaikh et al., 2009).

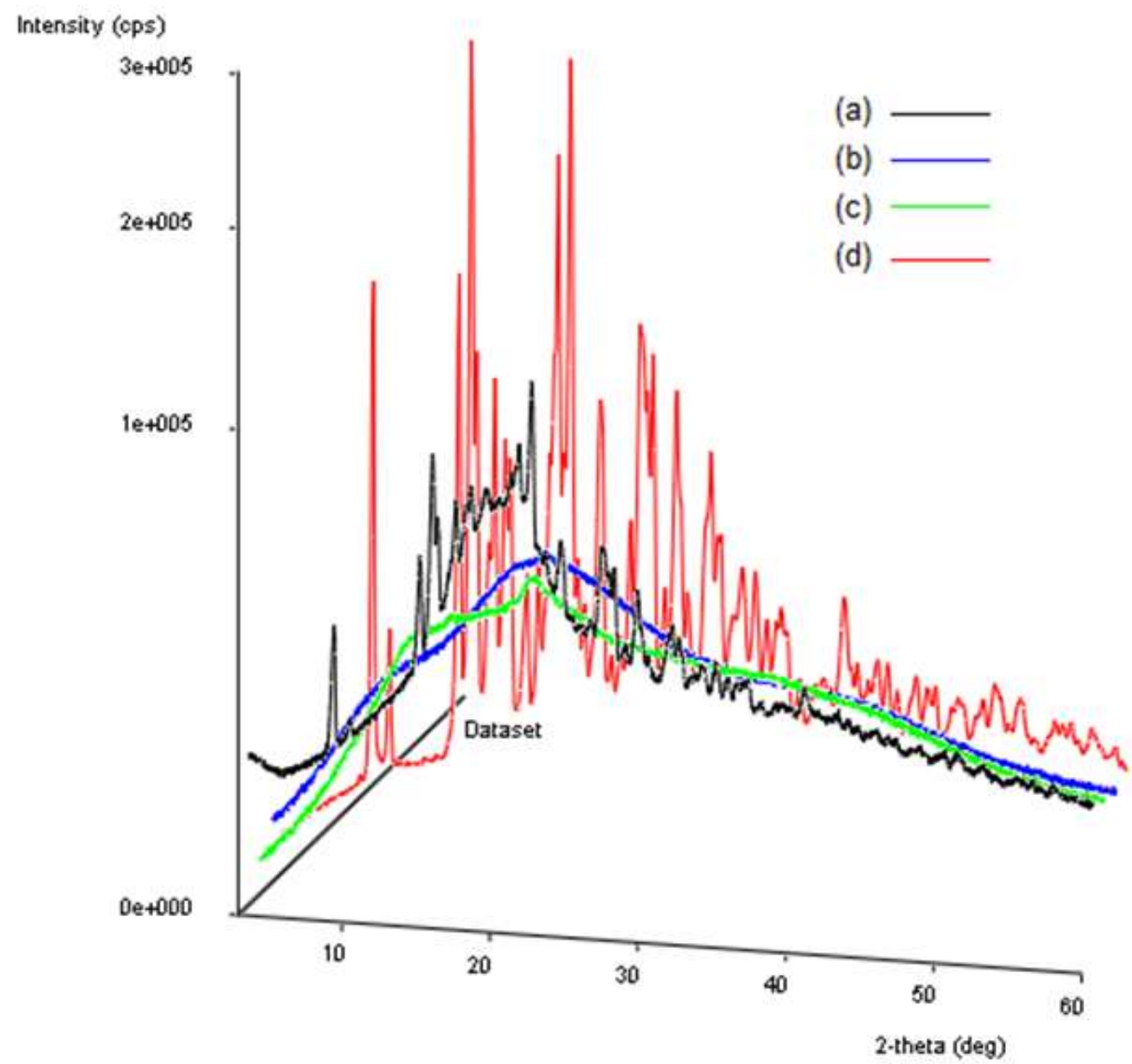

Fig. 8. Three dimensional X-ray diffractograms of; (a) AZT-loaded PEG-encl-PEC:MUC SMMs (black), pristine PEC (green), pristine MUC (blue) and pristine AZT (red). 


\subsection{In vitro drug release from PEG-encl-PEC:MUC SMMs}

The release of AZT from SMMs in SVF occurred mostly within 24 hours for the 15 BoxBehnken design formulations as depicted in the dissolution profiles in Fig. 9. The ability of the macromolecular SMMs to retain drug and prolong drug release was evaluated using the MDT. The MDT varied from 3.573 hours for formulation F3 to 6.300 hours for formulation F7 (Table 4). The optimized formulation had a MDT of 5.974 hours. Comparative drug release of the optimized formulation in PBS pH 7.4 yielded a MDT of 5.389 hours (Fig. 9d). Drug release from the optimized SMMs within 24 hours in SVF pH 4.5 was approximately $91 \%$ as compared to approximately $94 \%$ in PBS (Fig. 9d).
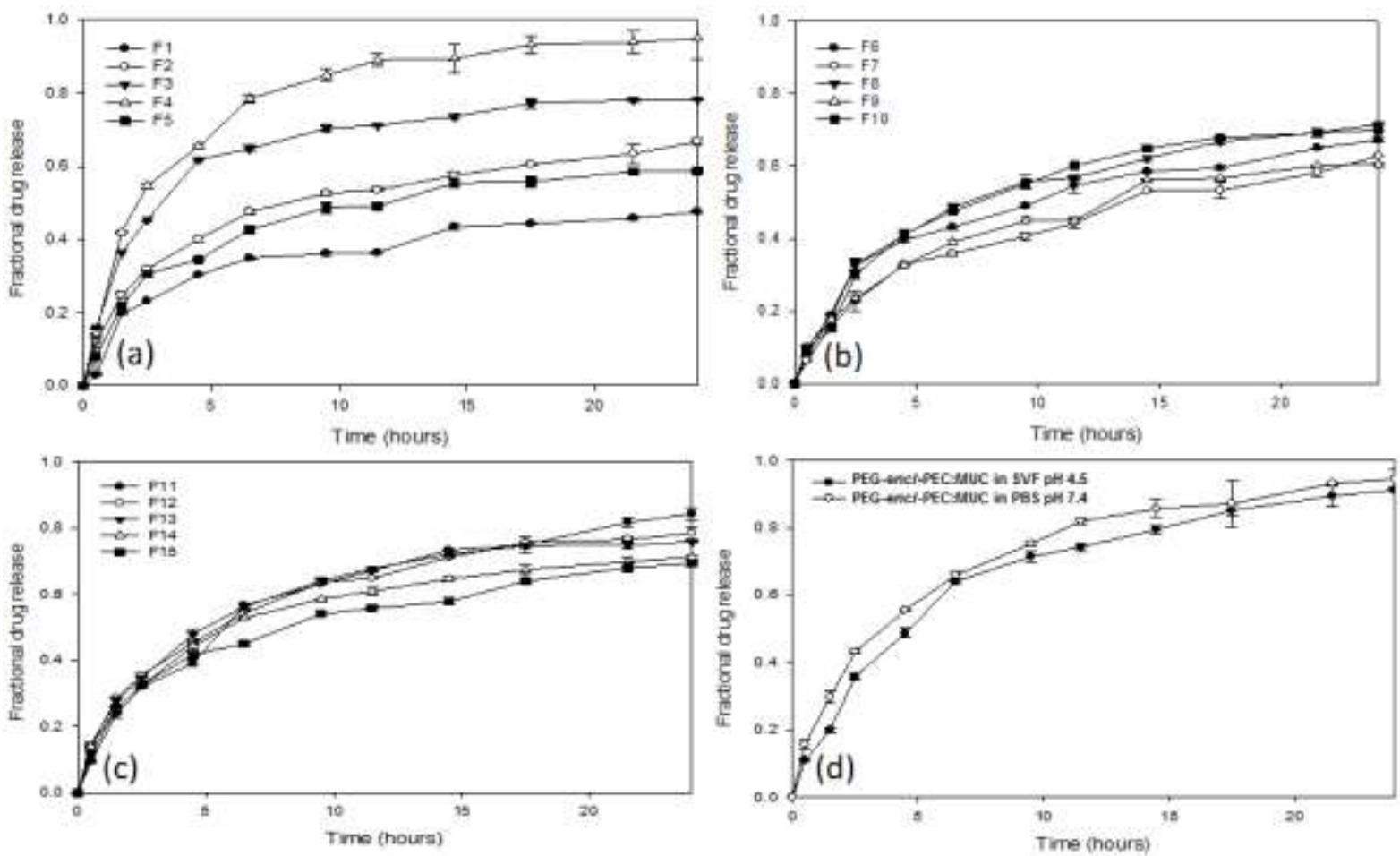

Fig. 9. SMM dissolution profiles of; (a) formulations F1-F5, (b) formulations F6-F10, (c) formulations F11-F15 and (d) the optimized formulation in SVF and PBS.

An increase in $\mathrm{pH}$ from acidic (pH 4.5 SVF) to alkaline (pH 7.4 PBS) might have caused a destabilization in the architecture of the SMMs leading to increased leakage of the encapsulated drug as shown by the increase in the fractional drug release in PBS as compared to that seen in SVF in Fig. 9d. Additionally, the mean dissolution time for the drug released from the SMMs was shorter in PBS as compared to SVF. This variation in drug release in dissolution media of different $\mathrm{pH}$ may be explained by the presents of carboxyl groups in pectin and mucin which are neutral in an acidic environment (SVF; pH4.5) and but ionized in an alkaline environment (PBS pH 7.4) which would lead to repulsion of the negatively charged moieties thus causing an architectural destabilization of the SMMs leading to increased drug release and a decreased mean dissolution time. However, the 
effect appeared not too pronounced possibly because pectin and mucin were interpenetrated and crosslinked. This was also confirmed by the similarity of the SMM dissolution profiles obtained in SVF and in PBS as specified by the closeness of their dissolution efficiencies (DE $=69.097 \%$ in SVF and DE=73.568\% in PBS) and according to the computed similarity and difference factors $\left(f_{2}=63.132\right.$ and $\left.f_{1}=8.099\right)$ (Moore and Flanner, 1996; O'Hara et al., 1998; Costa et al., 2001; Zhang et al., 2010).
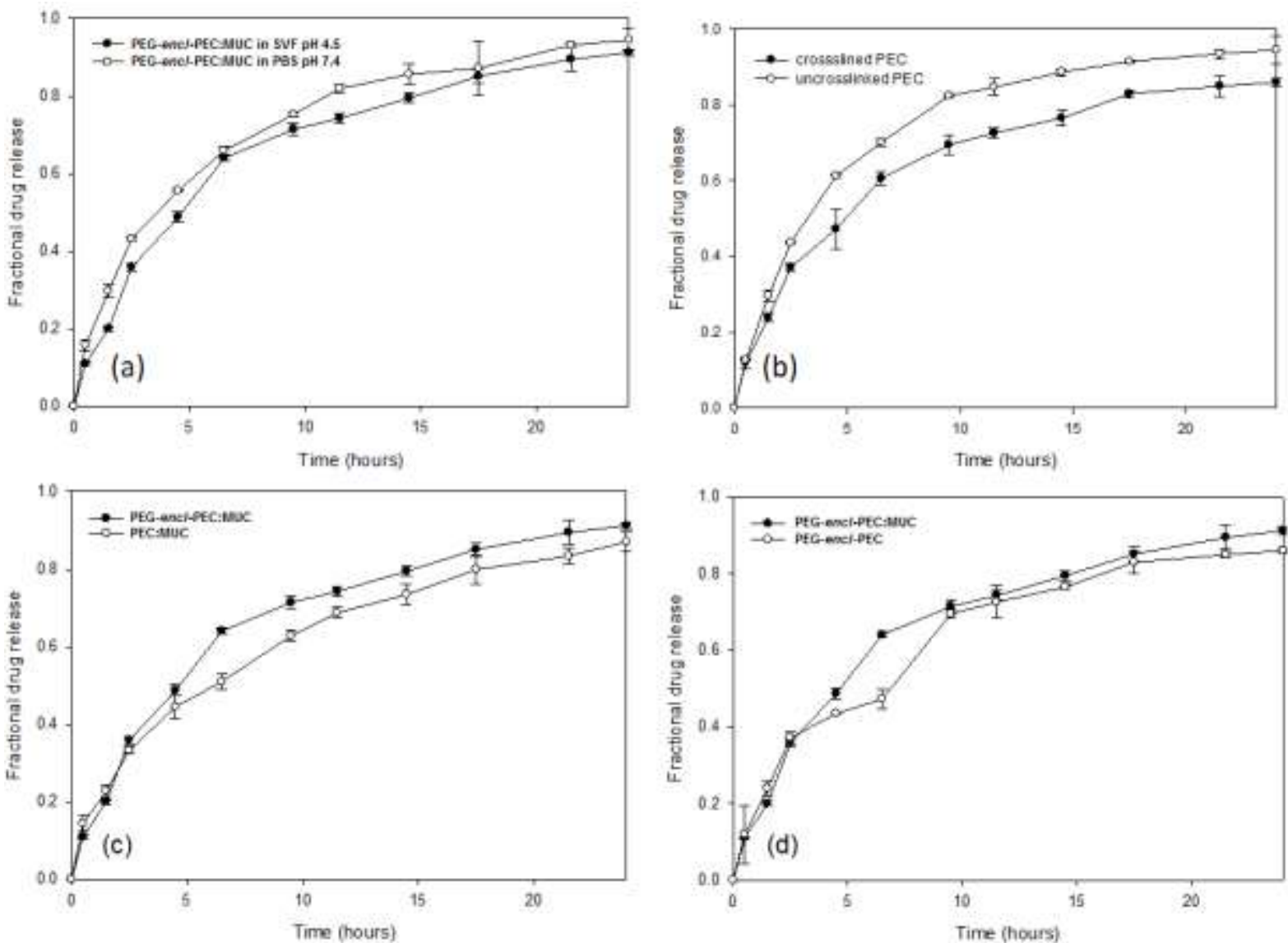

Fig. 10. Comparison of AZT release from; (a) PEG-encl-PEC:MUC SMMs in SVF pH 4.5 to AZT release in PBS pH 7.4, (b) crosslinked PEC SMMs to release from uncrosslinked PEC SMMs both in SVF, (c) PEGencl-PEC:MUC SMMs to AZT release from PEC-MUC SMMs both in SVF, and (d) PEG-enc/-PEC:MUC SMMs to AZT release in PEC-PEG SMMs both in SVF.

From the dissolution profiles in Fig. 10, it was noted that ionic crosslinking PEC with $\mathrm{Ca}^{2+}$ reduced the amount of drug released in 24 hours and this may have been a result of the formation of an egg-box network structure when the $\mathrm{Ca}^{2+}$ electrostatically interacted with the carboxylic moieties of the macromolecular PEC (Yu et al., 2009). This egg-box structure condenses the PEC chains encapsulating AZT leading to reduced dissolution. PEG caused a slight increase in the amount of drug released and this may have occurred because PEG draws water molecules towards the SMMs by forming hydrogen bonds to the surrounding water molecules from the dissolution media, SVF or PBS). 
Table 5: Goodness of fit and best fit parameters obtained by fitting the SMM dissolution profiles [obtained in SVF (pH4.5) and PBS (pH 7.4)] into different kinetic models

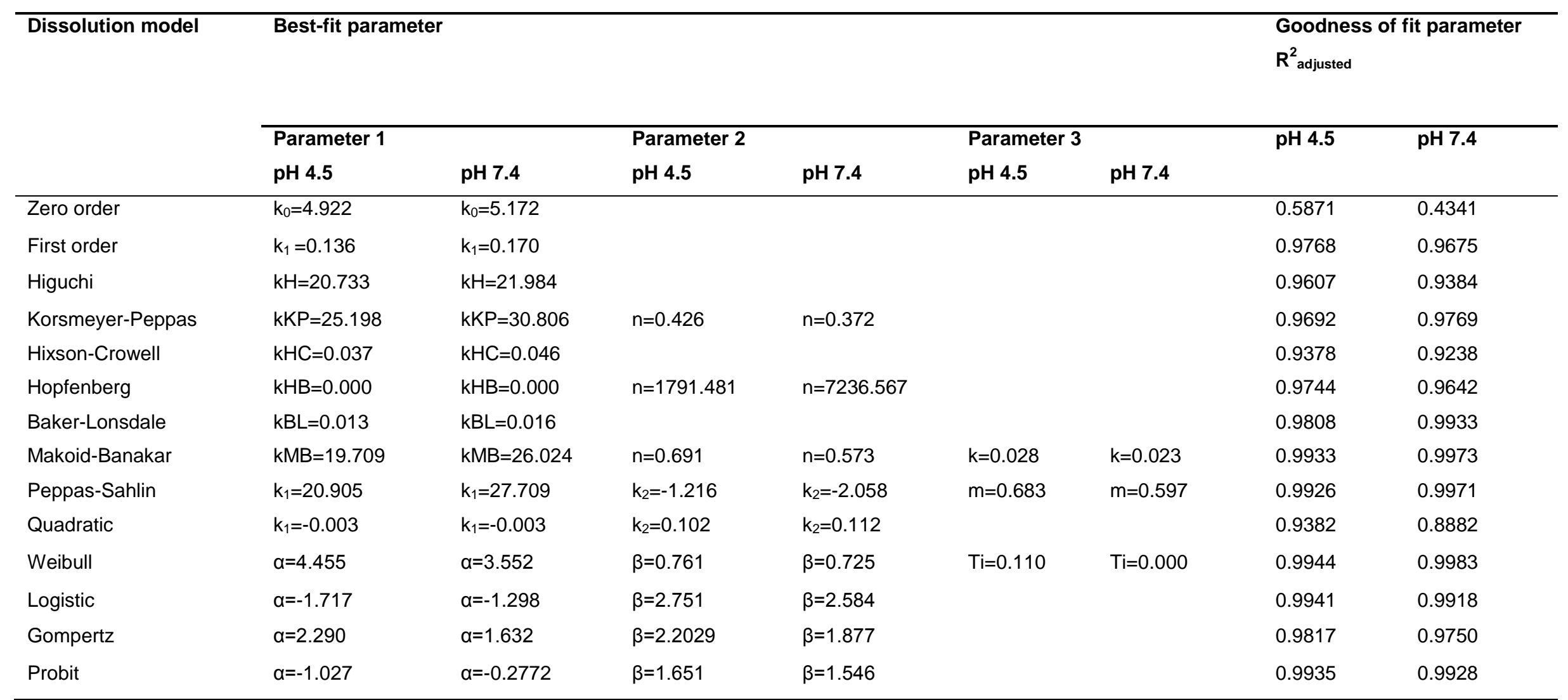

$\mathrm{k}_{0}, \mathrm{k}_{1}, \mathrm{kH}, \mathrm{kKP}, \mathrm{n}, \mathrm{kKP}, \mathrm{kHC}, \mathrm{kHB}, \mathrm{kBL}, \mathrm{k}, \mathrm{k}_{2}, \alpha$ and $\beta$ are best fit parameters of the respective dissolution models 
Modeling of the dissolution in SVF and PBS showed that AZT release kinetics fitted with most kinetics models tested as given by the values of the adjusted coefficient of determination ( $\mathrm{R}_{\text {adjusted }}$ ) with the exception of the zero order kinetic model as shown in Table 5. The highest goodness of fit parameter $\left(\mathrm{R}_{\text {adjusted }}^{2}\right)$ was attained using the Logistic and Makoid-Banakar models for the dissolution profiles obtained in SVF and PBS, respectively. The closeness of the best fit parameters and $R^{2}$ adjusted underline the similarity in the SMMs dissolution kinetics and profiles when dissolution was performed in SVF ( $\mathrm{pH} 4.5)$ as compared to dissolution in PBS (pH 7.4).

\section{Conclusions}

AZT-loaded PEG-encl-PEC:MUC submicron matrices were successfully formulated. A BoxBehnken design optimization was performed for the submicron matrices. The optimised submicron matrices prepared by a crosslinking-emulsion technique were spherical and not agglomerated. Drug release studies performed over 24 hours showed that approximately $91 \%$ and $94 \%$ of AZT was released in SVF and PBS (which acted as simulated semen), respectively. Drug release kinetics of the submicron matrices were found to fit most models tested with the highest goodness of fit parameter $\left(\mathrm{R}^{2}{ }_{\text {adjusted }}\right)$ being observed with the Logistic and Makoid-Banakar models for the dissolution profiles obtained in SVF and PBS, respectively.

\section{Acknowledgements}

This work was funded by the National Research Foundation (NRF) of South Africa.

\section{Conflict of Interest}

The authors confirm that there are no conflicts of interest.

\section{References}

Adams, J.L., Kashuba, D.M., 2012. Formulation, pharmacokinetics and pharmacodynamics of topical microbicides. Best Pract. Res. Clin. Obstet. Gynaecol. 26(4), 451-462.

Araújo, A.A.S., Storpirtis, S., Mercuri, L.P., Carvalho, F.M.S., dos Santos Filho, M., Matos, J.R., 2003. Thermal analysis of the antiretroviral zidovudine and evaluation of the compatibility with excipients used in solid dosage forms. Int. J. Pharm. 260, 303-314.

Box, G.E.P., Behnken, D.W., 1960. Some new three level designs for the study of quantitative variables. Technometrics 2, 455-475.

Celia, C., Trapasso, E., Cosco D., Paolino D., Fresta, M., 2009. Turbiscan Lab ${ }^{\circledR}$ Expert analysis of the stability of ethosomes ${ }^{\circledR}$ and ultradeformable liposomes containing a bilayer fluidizing agent. Colloids Surf. B: Biointerfaces 72, 155-160. 
Costa, P., Lobo, J.M.S., 2001. Modeling and comparison of dissolution profiles. Eur. J. Pharm. Sci. 13, 123-133.

Garcia, X., Escribano, E., Domenech, J., Quaeralt, J., Freixes, J., 2011. In vitro characterization and in vivo analgesic and anti-allodynic activity of PLGA-bupivacaine nanoparticles. J. Nanopart. Res. 13, 2213-2223.

Gupta, J., Tao, J.Q., Garg, S., Al-Kassas, R., 2011. Design and development of an in-vitro assay for evaluation of solid vaginal dosage forms. Pharm. Pharmacol. 2, 289-298.

Hendrix, C.W., Cao, Y.J., Fuchs, E.J. 2009. Topical microbicides to prevent HIV: clinical drug development challenges. Annu. Rev. Pharmacol. Toxicol. 49, 349-375.

Karnachi, A.A., Khan, M.A., 1996. Box-Behnken design for the optimization of variables of indomentacin coprecipitates with polymer mixtures. Int. J. Pharm. 131, 9-17.

Khan, K.A. 1975. The concept of dissolution efficiency. J. Pharm. Pharmacol. 27, 48-49.

Klaase, P.J., Shattock, R., Moore, J.P. 2008. Antiretroviral drug-based microbicides to prevent HIV-1 sexual transmission. Annu. Rev. Med. 59, 455-71.

Kumar, M., Mishra, R.K., Banthia, A.K., 2010. Development of pectin based hydrogel membranes for biomedical applications. Int. J. Plast. Technol. 14, 213-223.

Lemarchand, C., Couvreur, P., Vauthier, C., Costantini, D., Gref, R., 2003. Study of emulsion stabilization by graft copolymers using the optical analyzer Turbiscan. Int. J. Pharm. 254, 7782.

Mashingaidze, F., Choonara, Y.E., Kumar, P., du Toit, L.C., Maharaj, V., Buchmann, E., Ndesendo, V.M.K., Pillay, V., 2013. Exploration of the biomacromolecular interactions of an interpenetrating proteo-saccharide hydrogel network at the mucosal interface. J. Biomed. Mater. Res. Part A 101A, 3616-3629.

Mengual, O., Meunier, G., Cayre, I., Puech, K., Snabre, P., 1999. Characterisation of instability of concentrated dispersions by a new optical analyser: the TURBISCAN MA 1000. Colloids Surf. A: Physicochem. Eng. Aspects 152, 111-123.

Mishra, R.K., Datt, M., Pal, K., 2008. Banthia, A.K., Preparation and characterization of amidated pectin based hydrogels for drug delivery. J. Mater. Sci: Mater. Med. 19, 22752280.

Moore, J.W., Flanner, H.H., 1996. Mathematical comparison of dissolution profiles. Pharm.Tech. 1, 405-414.

O'Hara, T., Dunne, A., Butler, J., Devane, J., 1998. A review of methods used to compare dissolution profile data. Pharm. Sci. Technol. Today 1, 214-223.

Owen, D.H., Katz, D.F., 1999. A vaginal fluid simulant. Contraception. 59, 91-95.

Owen, D.H., Katz, D.F., 2005. A review of the physical and chemical properties of human semen and the formulation of a semen simulant. J. Androl. 26, 459-469.

Patel, M.M., Smart, J.D., Nevell, T.G., Ewen, R.J., Eaton, P.J., Tsibouklis, J., 2003. Mucin/poly(acrylic acid) interactions: a spectroscopic investigation of mucoadhesion. Biomacromolecules 4, 1184-1190. 
Pillay, V., Mashingaidze, F., Choonara, Y.E., du Toit, L.C., Buchmann. E., Maharaj, V., Ndesendo, V.M.K., Kumar, P., 2012. Qualitative and quantitative intravaginal targeting: key to anti-HIV-1 microbicide delivery from test tube to in vivo success. J. Pharm. Sci. 101(6), 1950-1968.

Ravi, P.R, Kotreka, U.K., Saha, R.N., 2008. Controlled release matrix tablets of zidovudine: effect of formulation variables on the in vitro drug release kinetics. AAPS PharmSciTech 9, 302-313.

Raviolo, M.A., Briñón, M., 2011. Preformulation studies of zidovudine derivatives: acid dissociation constants, differential scanning calorimetry, thermogravimetry, X-rays powder diffractometry and aqueous stability studies. Sci. Pharm. 79, 479-491.

Santos, C.A., Freedman, B.D., Ghosan, S., Jacob, J.S., Scarpulla, M., Mathiowitz, E., 2003. Evaluation of anhydride oligomer within polymer microsphere blends and their impact on bioadhesion and drug delivery in vitro. Biomaterials 24, 3571-3583.

Shaikh, J., Ankola, D.D., Beniwal, V., Sing, D., Ravi Kumar, M.N.V., 2009. Nanoparticle encapsulation improves oral bioavailability of curcumin by at least 9 -fold when compared to curcumin administered with piperine as absorption enhancer. Eur. J. Pharm. Sci. 37, 223230.

Stone, A., 2002. Microbicides: A new approach to preventing HIV and other sexually transmitted infections. Nat. Rev. Drug. Discov. 1, 977-985.

Woolfson, A.D., Umrethia, M.L., Kett, V.L., Malcolm, R.K., 2010. Freeze-dried, mucoadhesive system for vaginal delivery of the HIV microbicide, dapivirine: optimization by an artificial neural network. Int. J. Pharm. 388, 136-143.

Yu, C-Y., Cao, H., Zhang, X-C., Zhou, F-Z., Cheng S-X., Zhang X-Z., Zhuo, R-X., 2009. Hybrid nanospheres and vesicles based on pectin as drug carriers. Langmuir 25, 11720 11726.

Zhang, Y., Huo, M., Zhou, J., Zou, A., Li, W., Yao, C., Xie., S. 2010. DDSolver: An add-in program for modelling and comparison of drug dissolution profiles. AAPS J. 12, 263-271. 